\title{
Sistema Integrado de Informações Mais Médicos: uma ferramenta de suporte à gestão baseada em evidências
}

\author{
Carlos Rosales, ${ }^{1}$ Gabriel Vivas Francesconi, ${ }^{2}$ Joaquín Molina, ${ }^{2}$ Gabriel Listovsky, ${ }^{3}$ \\ Carolina Carvalho, ${ }^{1}$ Elisandréa Sguario Kemper, ${ }^{1}$ Viviane Santos Rocha ${ }^{1}$ e Thiago Augusto \\ Hernandes Rocha'
}

Como citar

Rosales C, Francesconi GV, Molina J, Listovsky G, Carvalho C, Kemper ES, et al. Sistema Integrado de Informações Mais Médicos: uma ferramenta de suporte à gestão baseada em evidências. Rev Panam Salud Publica. 2020;44:e65 https://doi.org/10.26633/RPSP.2020.65

RESUMO O presente trabalho busca detalhar uma experiência pioneira de desenvolvimento de um sistema de infor-
mação em saúde voltado para subsidiar a gestão com base em evidências junto à atenção primária à saúde
(APS). O processo de estruturação de programas e políticas públicas muitas vezes se dá sem acesso às
melhores evidências científicas. Nesse contexto, o Sistema Integrado de Informações Mais Médicos (SIMM),
aqui descrito, materializou um esforço para suprir a lacuna de informações para a gestão em saúde. Criado
com objetivo de integrar os dados das principais fontes de informação relativas ao Projeto de Cooperação
entre governo brasileiro e Organização Pan-Americana da Saúde/Organização Mundial da Saúde (OPAS/
OMS) para provimento de médicos no Programa Mais Médicos (PMM), o SIMM buscou otimizar a geração e
a consolidação de informações que possam subsidiar decisões, além de apoiar o controle administrativo e
financeiro. A experiência ilustra o desenvolvimento de uma solução auxiliar que pode ser ampliada para moni-
torar parâmetros de recursos humanos em saúde não apenas no PMM, mas na APS como um todo. A expertise
desenvolvida criou condições para que o sistema pudesse ser configurado como um bem público, para além
do suporte à gestão de um projeto em específico. O sistema pode ser adaptado e replicado em outros países
das Américas para melhorar a disponibilidade e a qualidade das informações utilizadas por tomadores de
decisão. mada por evidências; atenção primária à saúde.

\begin{abstract}
A estruturação de programas e políticas públicas em saúde é uma atividade complexa, marcada por múltiplos desafios. Usualmente, as intervenções em saúde carecem de um delineamento metodológico anterior à implementação, que permita o desenho de iniciativas de avaliação de desempenho. Os preceitos científicos associados a método, temporalidade e inferência causal nem sempre se fazem presentes durante o desenvolvimento de políticas e intervenções em saúde. Isso decorre de pressões que extrapolam o campo técnico-científico, mas que,
\end{abstract}

ainda assim, são capazes de modelar a estrutura de implantação de uma política: a divergência entre os tempos da gestão e da academia pode contribuir para que decisões críticas, em situações de pressão, sejam tomadas sem o devido suporte de evidências válidas (1). De fato, observa-se uma baixa utilização de resultados concretos, obtidos a partir de análises empíricas, para subsidiar uma gestão baseada em evidências (2). Assim, aproximar esses tempos é uma via possível para instrumentalizar decisões de políticas em saúde. Para tanto, são

\footnotetext{
Organização Pan-Americana da Saúde (OPAS), Unidade Técnica Mais Médicos, Brasília (DF), Brasil. $\bowtie$ Thiago Augusto Hernandes Rocha, rochahernandes3@ gmail.com
}

\footnotetext{
2 Organização Pan-Americana da Saúde (OPAS), Brasília (DF), Brasil. 3 Organización Panamericana de la Salud (OPS), Cidade do Panamá Panamá.
} 
essenciais ações voltadas para a produção célere e efetiva de informações.

A gestão baseada em evidências pode ser compreendida como o uso consciente, criterioso, do melhor conjunto de informações disponíveis para a definição da melhor estratégia orientada para a resolução de um problema (3). Esse conceito, originado nos preceitos da medicina baseada em evidências, tem sido amplamente aplicado em gestão.

O contexto da saúde pública não é diferente. Para que seja possível instrumentalizar gestores para a tomada de decisão com base em informações, potencialmente melhorando condições de saúde e diminuindo desigualdades, é fundamental indagar que tipo de evidência se faz necessária para apoiar as ações (4). Mesmo a evidência mais sólida se torna sem efeito caso não seja incorporada à rotina de tomada de decisões (5). Estudos recentes destacam que há poucas iniciativas voltadas para avaliação de práticas de implementação (2). Superar esse entrave envolve a participação de gestores e tomadores de decisão em todos os momentos, desde os estágios iniciais do desenvolvimento de políticas em saúde. Assim, é possível mapear as lacunas a serem tratadas e traduzir as evidências em práticas concretas $(1,6)$.

Há uma crescente literatura que discute as barreiras à utilização de evidências para subsídio à gestão. Andermann et al. (1) apontam quatro grandes tópicos que sumarizam as principais dificuldades para viabilizar uma gestão baseada em evidências: 1) perda de janelas de oportunidade, 2) lacunas de conhecimento e incertezas, 3) evidências controversas ou conflitantes e 4) conflitos de interesse. Essas barreiras surgem em decorrência da complexidade inerente à produção de evidências científicas e de conhecimento, da desconexão entre o mundo da academia e aquele dos formuladores de políticas e da subversão de evidências para a promoção de ganhos políticos ou econômicos (1). A superação dessas questões demanda a disponibilização de informações em tempo e formato adequados (7).

No escopo da saúde, os sistemas de informação em saúde (SIS) são definidos como um conjunto de componentes interrelacionados que coletam, processam, armazenam e distribuem informações a fim de apoiar o processo decisório e auxiliar na organização do sistema de saúde (8). Assim, espera-se que esses sistemas instrumentalizem gestores com evidências. Entretanto, apesar de seu potencial, poucas iniciativas voltadas para o monitoramento e a produção de evidências amparadas em SIS têm sido desenvolvidas (8). A maior parte dos SIS utilizados são voltados a aspectos clínicos, dispensando uma interface mais próxima à gestão $(9,10)$.

Buscando suprir essa lacuna, a Organização Pan-Americana da Saúde/Organização Mundial da Saúde (OPAS/OMS) tem fomentado iniciativas de melhoria dos SIS (11) nas Américas, com o intuito de incentivar o desenvolvimento de soluções que permitam transformar o conhecimento científico em subsídio para decisões amparadas em dados. Entretanto, não foram reportadas iniciativas dedicadas à geração de evidências para permitir o monitoramento e acompanhamento de iniciativas no âmbito da atenção primária à saúde (APS). Nesse sentido, o presente trabalho busca detalhar a experiência pioneira de desenvolvimento do Sistema Integrado de Informação Mais Médicos (SIMM). Esse sistema foi criado para preencher a lacuna referente à necessidade de geração de dados e informações para subsidiar o monitoramento e a avaliação do Programa Mais Médicos (PMM) no Brasil.

\section{CONTEXTO DE ESTRUTURAÇÃO DO SISTEMA INTEGRADO DE INFORMAÇÕ̃ES MAIS MÉDICOS}

O PMM foi instituído no Brasil em 2013 tendo como objetivo principal aumentar a disponibilidade de profissionais médicos no Sistema Único de Saúde (SUS), principalmente em áreas geográficas onde o número de médicos por habitante era baixo $(12,13)$. O PMM contemplou três eixos: mudança na matriz curricular dos cursos de medicina, com respectiva ampliação de vagas; reaparelhamento das unidades básicas de saúde; e provimento emergencial de médicos brasileiros (formados ou não no exterior) e estrangeiros por um período de 3 anos (prorrogável por mais 3) em localidades com escassez de profissionais (12). Vale ressaltar que foram priorizados, na ocupação das vagas, os médicos formados em instituições brasileiras de ensino superior ou com diplomas revalidados, considerados médicos participantes, seguidos de médicos brasileiros formados em instituições estrangeiras com habilitação para exercício da medicina no exterior. Num momento posterior, foram disponibilizadas vagas para médicos estrangeiros, sendo esses chamados de médicos intercambistas. Nessa categoria foram inseridos os médicos providos por meio do Projeto de Cooperação Mais Médicos com a OPAS/OMS. Até meados de 2015, havia aproximadamente 18240 profissionais atuando no PMM em $81 \%$ dos municípios brasileiros, incluindo todas as categorias de médicos. Também estava prevista a criação de 11,5 mil novas vagas de graduação em medicina no país (14).

Os três eixos do PMM, além de agregar medidas emergenciais de provisão de médicos a partes de populações até então desassistidas, se propunham a modificar, em médio e longo prazo, a estrutura de provimento e formação de médicos no Brasil. Ao reestruturar a forma como são alocados os profissionais e alterar a lógica de formação médica, o PMM colocou a provisão de médicos para APS no centro da discussão política em saúde do país. Além disso, atribuiu à gestão de recursos humanos em saúde um papel norteador da política de APS brasileira $(13,15,16)$.

As complexidades inerentes ao PMM exigiram a estruturação de sistemas de informação específicos, capazes de instrumentalizar a gestão para a tomada de decisão e promover o monitoramento do grande volume de operações necessárias para o seu funcionamento. Entre os sistemas utilizados como ferramenta de monitoramento e gestão do PMM pelo Ministério da Saúde, vale referir o Sistema de Gerenciamento de Programas (SGP), que se encontra sob responsabilidade da Secretaria de Gestão do Trabalho e da Educação na Saúde (SGTES/MS) (17). Por meio dele, gestores municipais e profissionais médicos realizam as adesões e inscrições nos programas de provimento de médicos do Governo Federal e registram suas informações de cadastro. O SGP também é capaz de acessar dados atualizados do Sistema de Cadastro Nacional de Estabelecimentos de Saúde (SCNES) e, a partir disso, ofertar informações relacionadas à lotação de médicos atualizadas mensalmente.

Por parte da OPAS/OMS, os sistemas inicialmente utilizados para fins de monitoramento do Projeto de Cooperação eram o Sistema para la Cooperación Médica (COLPADI) e o Pan American Sanitary Bureau Management Information System (PMIS). O primeiro era utilizado para controle das ações de registro e movimentação dos médicos inseridos no Projeto, ao passo que o segundo era voltado ao controle administrativo-financeiro. Entretanto, o propósito de cooperar tecnicamente com o Brasil 
através do provimento emergencial de profissionais médicos, do intercâmbio internacional de conhecimentos e da produção de cuidado envolveu manejo de um grande volume de operações, o que impôs a necessidade de um SIS que fosse capaz de agregar as diferentes nuances do Projeto de Cooperação Técnica.

Dessa necessidade surgiu o SIMM, desenvolvido pela OPAS em parceria com o Campus Virtual de Saúde Pública (CVSP) / Panamá - com o objetivo de integrar os dados das principais fontes de informação sobre o Projeto de Cooperação Mais Médicos, subsidiar os processos de tomada de decisão e apoiar o controle administrativo e financeiro. $\mathrm{O}$ estabelecimento de estruturas de monitoramento capazes de evidenciar a evolução de indicadores-chave de desempenho é fundamental para o delineamento de um ambiente de gestão propício à tradução de objetivos estratégicos em ação (18). Considerando essa premissa, o SIMM buscou suprir algumas lacunas de acompanhamento de evolução do PMM que antes não podiam ser adequadamente abordadas em função da pulverização de informações em diferentes bases de dados.

O SIMM articula uma plataforma virtual para o monitoramento do PMM por meio de um aplicativo, disponível para desktop, iOS e Android. O sistema introduziu diversas funcionalidades, como coleta dados, canal de comunicação com os médicos cooperados cubanos inseridos nas atividades de ensino-serviço do PMM (canal que não é mais utilizado em função do regresso desses médicos a Cuba em dezembro de 2018) e disponibilização de informações sobre o PMM para o público em geral. O sistema criou ainda uma área restrita voltada para as ações de controle e gestão do Projeto de Cooperação Mais Médicos. As informações disponibilizadas na área restrita têm sido utilizadas para o delineamento de ações de monitoramento e avaliação do PMM.

O SIMM foi desenvolvido utilizando um sistema de gestão de conteúdos denominado Drupal, o qual permite criar e organizar conteúdo, manipular a aparência, automatizar tarefas administrativas e definir permissões e papéis para usuários e colaboradores. Além disso, há a integração com painéis de monitoramento baseados em Tableau. Para o desenvolvimento do SIMM, foi fundamental a parceria com o CVSP/Panamá. O CVSP/Panamá aglutina uma rede de colaboradores, instituições e organizações que compartilham cursos, recursos, serviços e atividades de educação, informação e gestão do conhecimento em ações de formação, com o objetivo comum de aprimorar as habilidades da força de trabalho e as práticas de saúde pública (19). Para isso, o CVSP prioriza a inovação nas tecnologias de informação e comunicação, visando a melhoria contínua no desempenho de programas de educação permanente em saúde (19).

O CVSP/Panamá desenvolveu, ao longo dos últimos anos, grande expertise na elaboração de soluções e SIS nas Américas. Fundado em 2000 e tendo o escopo de atuação pautado pela cooperação técnica, o CVSP vem acumulando conhecimento que permite o estabelecimento de parcerias estratégicas para a potencialização de iniciativas voltadas para a gestão baseada em evidências (20). A parceria entre o CVSP/Panamá e a Unidade Técnica Mais Médicos (UTMM), responsável pela gestão do PMM na OPAS/OMS Brasil, foi sinérgica, o que permitiu a estruturação de um SIS capaz subsidiar com evidências a gestão do Projeto de Cooperação.

\section{ESTRUTURAÇÃO E FUNCIONALIDADES DO SIMM}

O SIMM integra os principais bancos de dados do PMM para o Brasil, trazendo informações sobre médicos e municípios nos quais estão inseridos especificamente médicos cubanos. O sistema está estruturado para atender diferentes tipos de usuário, com funcionalidades que variam de acordo com o perfil de acesso. Os três principais perfis de acesso são: público em geral, médico e administrador OPAS/OMS (subdividido em assessores nacionais e estaduais do Programa de Cooperação, gestor e administrador).

O perfil de acesso público refere-se a usuários em geral que optam por baixar o aplicativo do sistema no celular ou acessar os conteúdos via web (https://simm.campusvirtualsp.org/). Esses usuários podem ter acesso aos menus "Projeto de Cooperação Mais Médicos", "Painel de Informação" e "Resultados e Evidências" (figura 1). Os conteúdos desses menus trazem informações gerais sobre o PMM e sobre quantitativo, distribuição e perfil dos municípios com médicos cooperados. Além disso, o perfil de acesso público disponibiliza o conjunto de estudos e pesquisas sobre o PMM.

Entre todos os perfis, o perfil de administrador OPAS, utilizado pelos responsáveis pela gestão do Projeto de Cooperação, oferece as possibilidades mais amplas: esses usuários podem modificar a estrutura do sistema, controlar a postagem e a divulgação de informações e gerenciar a atribuição de papéis a outros usuários.

A figura 2 mostra as funcionalidades disponíveis para o perfil de administrador OPAS em sua área restrita. O menu "Meu Consultório" permite atualizar as informações cadastrais dos médicos, responder enquetes divulgadas pela UTMM e visualizar conteúdos de capacitação voltados para os médicos cooperados. Os perfis não enquadrados como administrador OPAS apresentam, em termos de possibilidades, uma parte das funcionalidades atribuídas àquele perfil.

Entre as funções do SIMM está o registro de correções necessárias nas informações referentes aos médicos cooperados. Apesar de não permitir a inserção de novas informações no COLPADI, o SIMM é capaz de manter um histórico das informações que precisaram ser corrigidas, em função de inconsistências observadas durante o manejo cotidiano das atividades do PMM. Esse menu também concede acesso às enquetes regulares disponibilizadas pela UTMM para fins de monitoramento e avaliação de aspectos relevantes do programa. As enquetes são diversas e atendem aos requisitos de monitoramento da UTMM, compreendendo temas como condições de trabalho e avaliação de conhecimentos dos médicos cooperados sobre temas de interesse epidemiológico do país.

O menu de acesso referente ao perfil administrador OPAS traz, ainda, informações internas sobre o projeto para fins de gestão do PMM. Esses dados compreendem elementos sobre a gerência da UTMM, acompanhamento de ações judiciais, monitoramento e avaliação do programa e painel de controle dos consultores internacionais (international professional consultants, ou IPC). Entre os indicadores disponibilizados na área restrita, destacam-se o número de médicos envolvidos em ações de especialização, a distribuição geográfica de profissionais, o número de médicos atuando na APS, o volume de recursos financeiros repassados e executados e o monitoramento de médicos ativos, baixas e intercorrências. 
FIGURA 1. Tela inicial do Sistema Integrado de Informações Mais Médicos para público geral em português, 2019

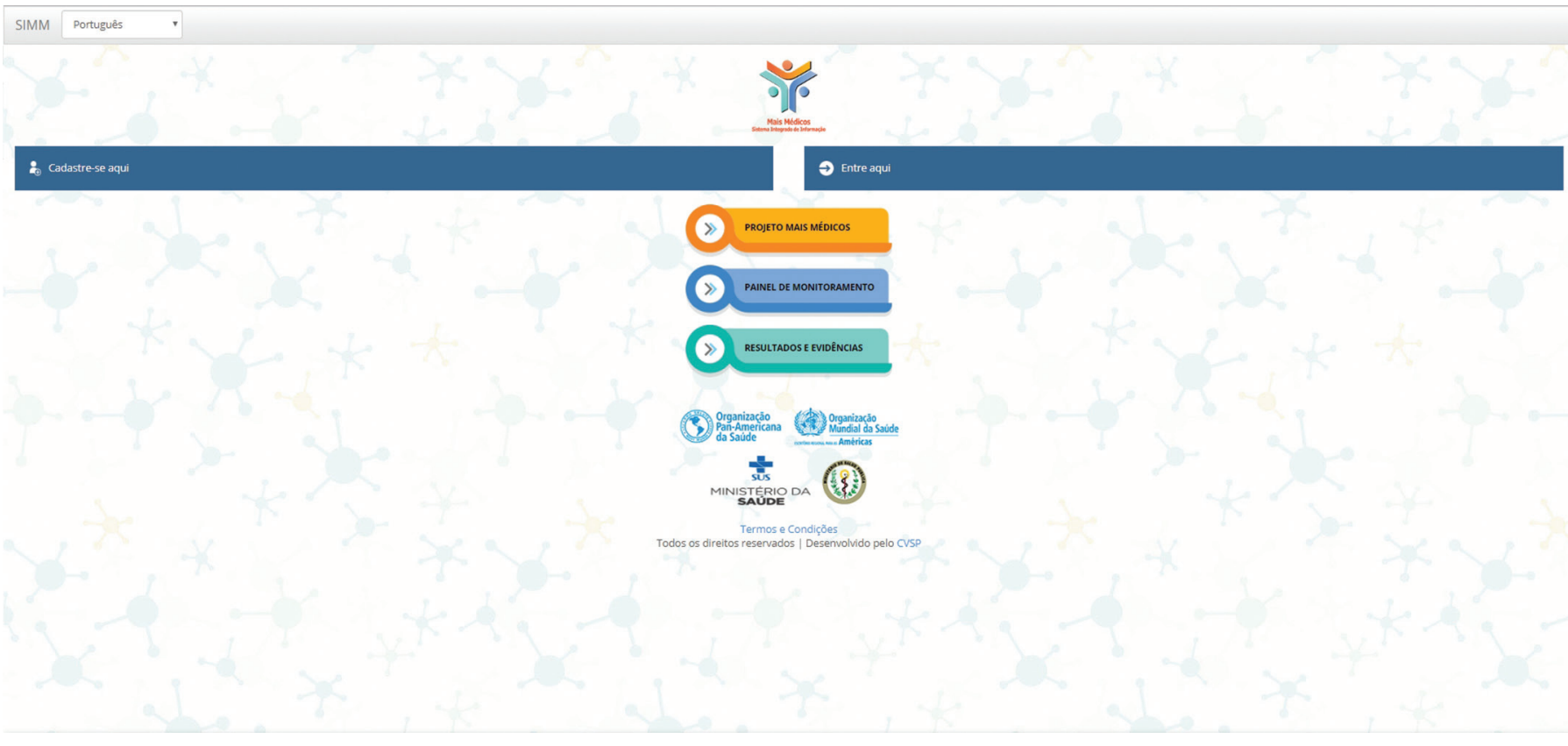

FIGURA 2. Tela inicial do perfil administrador, Sistema Integrado de Informações Mais Médicos, 2019

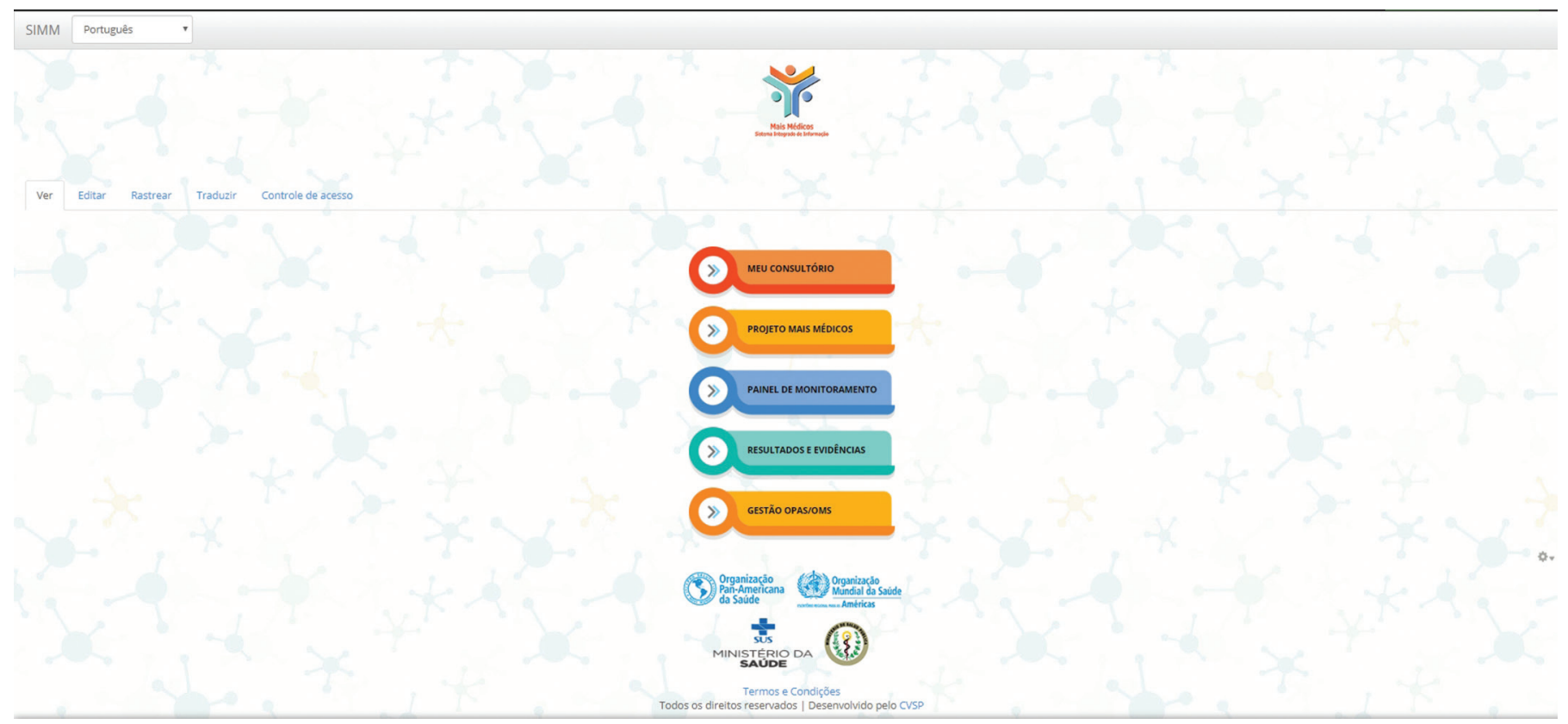

\section{POTENCIALIDADES E PERSPECTIVAS DO SIMM}

A importância da estruturação dos SIS resulta de sua potencialidade em melhor qualificar as decisões que são tomadas. Instrumentalizar essas decisões com evidências sólidas, capazes de estimar possíveis impactos das ações elencadas como prioritárias, contribui para a efetivação dos princípios de acesso universal à saúde. Nesse sentido, a experiência aqui detalhada ilustra como, a partir da identificação de uma lacuna, foi possível desenvolver uma solução capaz de subsidiar o processo decisório com evidências empíricas.
A presente iniciativa, voltada ao monitoramento de aspectos adjacentes ao projeto de cooperação técnica no escopo do PMM, pode ser ampliada com base na tecnologia já desenvolvida para permitir o monitoramento e o acompanhamento de parâmetros de recursos humanos em saúde. Tal monitoramento poderia ser direcionado não somente para o PMM, mas para a APS como um todo. A expertise desenvolvida criou condições para potencializar os benefícios da solução concebida, de forma a configurar essa solução como um bem público, e não somente como uma ferramenta de suporte à gestão de um projeto específico. 
FIGURA 3. Visualização de dados do SIMM com painéis de controle integrados mostrando informações estratégicas do Programa Mais Médicos, 2019

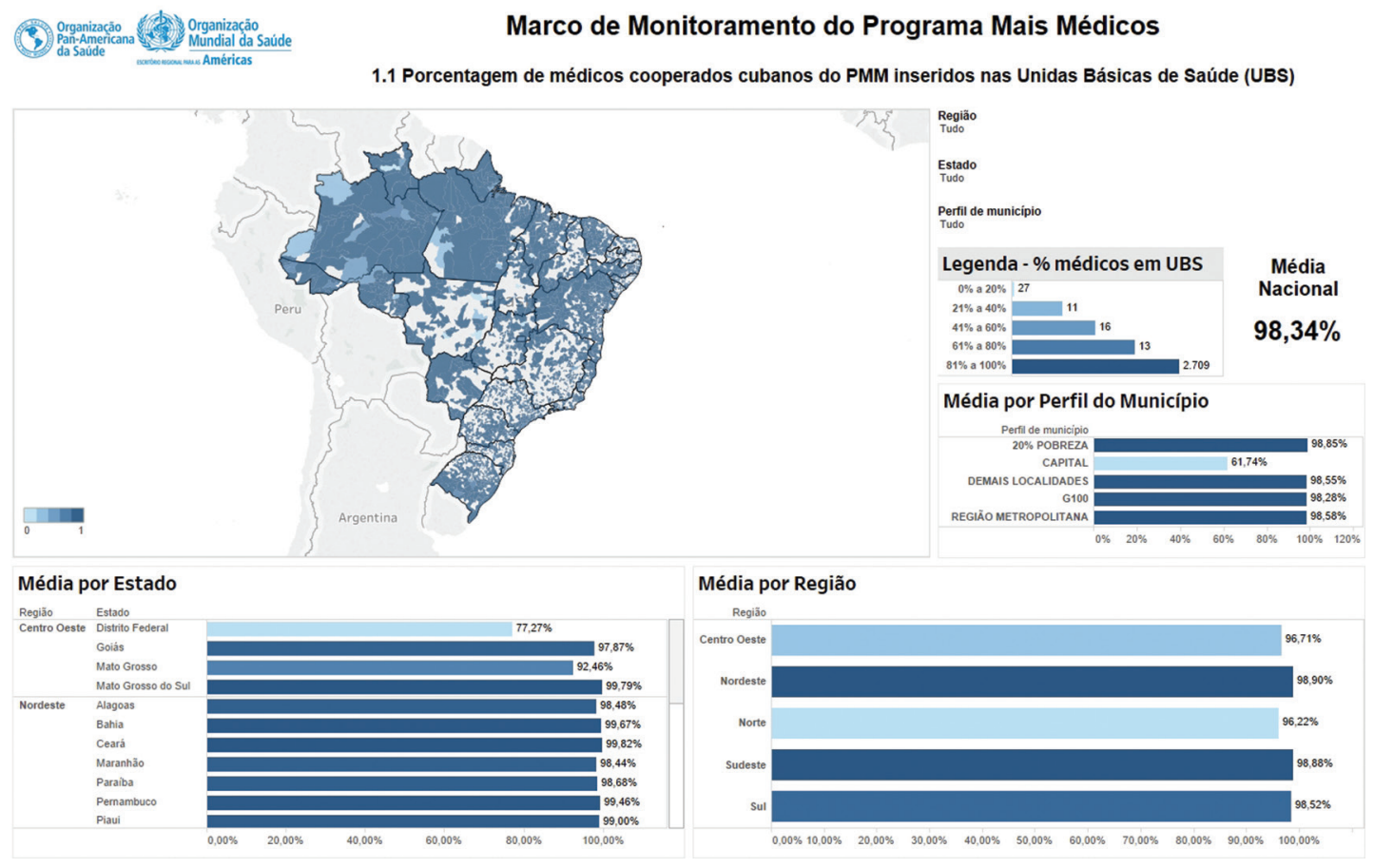

A saúde utiliza mão de obra em caráter intensivo. Em função disso, o monitoramento de elementos vinculados à dimensão de recursos humanos é fundamental para garantir a efetividade dos serviços ofertados à população. O SIMM é um exemplo de iniciativa que almejou instrumentalizar gestores envolvidos no manejo da APS com dados sobre aspectos de recursos humanos em saúde. Diante de sua potencialidade e dos benefícios produzidos, seria desejável sua expansão para outras esferas, não necessariamente adscritas ao PMM.

Considerando a lacuna existente, discutida ao longo do presente trabalho, um esforço de reformulação e ampliação do SIMM vem sendo conduzido. Com o apoio de especialistas em otimização de valor de soluções web do núcleo de Telessaúde da Universidade Federal do Rio Grande do Sul (TelessaúdeRSUFRGS), o SIMM vem sendo reformulado com melhorias na usabilidade, estrutura de navegação e veiculação de informações, de modo a potencializar o valor agregado aos seus usuários.

Ainda, a inserção de novos parâmetros para monitoramento da APS poderia ampliar a estrutura que vem sendo desenvolvida, de modo a incluir novos indicadores de recursos humanos em saúde não somente para o Brasil, mas para as Américas como um todo. Considerando que a OPAS vem trabalhando em uma proposta para o monitoramento da APS nas Américas, alinhada à Agenda para a Atenção Primária 30-30-30 (21), a tecnologia desenvolvida para o SIMM poderia servir como ponto de apoio para conferir rapidez ao processo de disponibilização de dados úteis à gestão para os diferentes países das Américas. $\mathrm{O}$ exemplo da figura 3 demonstra a integração de soluções para processamento e visualização de dados no SIMM, com suporte da utilização de painéis de controle em Tableau para gerar informações estratégicas sobre indicadores da APS. Tais indicadores poderiam ser estruturados para cobrir aspectos essenciais ao processo de tomada de decisões em saúde.
Desse modo, gestores, formuladores de políticas e pesquisadores em saúde poderiam se beneficiar de evidências para balizar suas decisões, contribuindo para a minimização de riscos e a melhoria do processo de oferta de serviços de saúde. Portanto, os passos trilhados na construção do SIMM podem se configurar como benchmark para outras iniciativas do gênero.

Entre as principais vantagens do SIMM destacam-se a integração de múltiplas fontes de dados em um único repositório, a forma de apresentação multiplataforma com ênfase em aplicativo mobile e a disponibilização de dados de monitoramento com o uso de painéis de controle dinâmicos. Essas características agregadas não foram encontradas em sistemas similares voltados à gestão de projetos da APS. Assim, o SIMM se configura como uma iniciativa pioneira nesse tema.

Apesar dos avanços, o SIMM ainda carece de melhorias em termos de funcionalidade e organização, como aquelas que vêm sendo implementadas com o apoio do núcleo de TelessaúdeRSUFRGS. Entre tais melhorias, destacam-se a maior capacidade de agregar valor, a navegação facilitada e o design aprimorado. As funcionalidades do SIMM e seu modus operandi podem ser replicados para outros países das Américas, a fim de monitorar ações de saúde e oferecer suporte a decisões com base em evidências.

Contribuição dos autores. CR, GVF, JM, GL, CC, ESK, VSR e TAHR contribuíram com a elaboração do documento, análise dos dados, redação do manuscrito e validação final.

Conflitos de interesse. Nada declarado pelos autores.

Declaração. As opiniões expressas no manuscrito são de responsabilidade exclusiva dos autores e não refletem necessariamente a opinião ou política da RPSP/PAJPH ou da Organização Pan-Americana da Saúde (OPAS). 


\section{REFERÊNCIAS}

1. Andermann A, Pang T, Newton JN, Davis A, Panisset U. Evidence for Health II: overcoming barriers to using evidence in policy and practice. Health Res Policy Syst. 2016;14(1):17.

2. Andermann A, Pang T, Newton JN, Davis A, Panisset U. Evidence for Health I: producing evidence for improving health and reducing inequities. Health Res Policy Syst. 2016;14(1):18.

3. Pfeffer J, Sutton RI. Evidence-based management. Harv Bus Rev. 2006;84(1):62-74,133.

4. Andermann A. Evidence for health: from patient choice to global policy. Cambridge: Cambridge University Press; 2012.

5. Lehmann U, Gilson L. Action learning for health system governance: the reward and challenge of co-production. Health Policy Plan. 2015;30(8):957-63.

6. Blalock AB. Evaluation research and the performance management movement: from estrangement to useful integration? Evaluation. 1999;5(2):117-49.

7. Gray JAM. Evidence-based Health Care. Edimburgo: Churchill Livingstone; 2001.

8. Rocha TAH, Rocha J, Silva N, Amaral P, Facchini L, Thumé E, et al. Cadastro nacional de estabelecimentos de saúde: evidências sobre a confiabilidade dos dados. Cienc Saude Colet. 2017;23(1):229-40.

9. Sandiford P, Annett H, Cibulskis R. What can information systems do for primary health care? An international perspective. Soc Sci Med. 1992;34(10):1077-87.

10. Chapman SR. Prescribing information systems: making sense of primary care data. J Clin Pharm Ther. 2001;26(4):235-9.

11. Pan American Health Organization (PAHO). Information systems for health (IS4H). Washington: PAHO; 2018. Disponível em: https: / / www.paho.org/ish/index.php/en/ Acessado em abril de 2019.

12. Carvalho VKS, Marques CP, da Silva EN. A contribuição do Programa mais médicos: análise a partir das recomendações da OMS para provimento de médicos. Cienc Saude Colet. 2016;21(9):2773-84.

13. Barbosa ACQ, Amaral PV, Francesconi GV, Rosales C, Kemper ES, da Silva NC, et al. Programa mais médicos: como avaliar o impacto de uma abordagem inovadora para superação de iniquidades em recursos humanos. Rev Panam Salud Publica. 2018;42:e185.
14. Campos GWS, Pereira Júnior N. A atenção primária e o Programa Mais Médicos do Sistema Único de Saúde: conquistas e limites. Cienc Saude Colet. 2016;21(9):2655-63.

15. Carrillo B, Feres J. More doctors, better health? Evidence from a physician distribution policy. Nova Iorque: Health, Econometrics and Data Group (HEDG); 2017.

16. Carrillo B, Feres J. Provider Supply, utilization, and infant health: evidence from a physician distribution policy. Am Econ J. 2018. Disponível em: https://www.aeaweb.org/articles?id=10.1257/pol $.20170619 \& \&$ from $=$ f Acessado em abril de 2019.

17. Brasil, Ministério da Saúde. Sistema de Gerenciamento de Programas. Brasília: Ministério da Saúde; 2014. Disponível em: http:// maismedicos.saude.gov.br/loginExt.php?denied=true Acessado em abril de 2019.

18. Bernus P, Błażewicz J, Schmidt GJ, Shaw MJ. International handbooks on information systems. Berlim: Springer; 2007.

19. Pan American Health Organization/World Health Organization (PAHO/WHO). Campus Virtual de Salud Publica. PAHO/WHO: Washington; 2008. Disponível em: https://www.campusvirtualsp .org/ Acessado em abril de 2019.

20. Cho M, Moya J, Hernández T, Flores M, Ledezma Y, Samán L, et al. Desarrollo del nodo Venezuela en el campus virtual de salud pública. Rev Panam Salud Publica. 2018;42:e71.

21. Pan American Health Organization (PAHO). Universal health in the 21st century: 40 years of Alma-Ata. Report of the High-Level Commission. Washington, DC: PAHO; 2019. Disponível em: http://iris .paho.org/xmlui/handle/123456789/50742 Acessado em maio de 2019

Manuscrito recebido em 12 de janeiro de 2019. Aceito em versão revisada em 9 de abril de 2019

\section{More Doctors Integrated Information System: a tool to support evidence-based management}

ABSTRACT

Keywords
The present work provides details about a novel experience involving the development of a health information system focused on supporting evidence-based management at the primary health care (PHC) level. The process of structuring public health programs and policies is often developed without access to the best available scientific evidence. In this context, the proposed system (Sistema Integrado de Informações Mais Médicos, SIMM) materializes the effort to bridge the information gap for health management. Created with the aim of integrating data from the main information sources associated with the Cooperation Project between the Brazilian federal government and the Pan American Health Organization/World Health Organization (PAHO/ WHO) for provision of physicians for the More Doctors Program, SIMM strove to optimize the generation and consolidation of information to support decision-making, in addition to providing support for administrative and financial control. The experience illustrates the development of an ancillary solution that can be expanded to monitor human resources for health parameters across the entire PHC setting, far beyond the More Doctors Program. The resulting expertise created conditions for the system to be configured as a public asset, rather than being restricted to providing managerial support for a specific project. SIMM may be adapted and replicated in other American countries to improve the availability and quality of the information used by decision makers.

Information systems; management information systems; information dissemination; evidence-informed policy; primary health care. 


\section{Sistema integrado de información de Más Médicos: una herramienta de apoyo a la gestión basada en la evidencia}

RESUMEN En el presente trabajo se detalla una experiencia pionera de desarrollo de un sistema de información de salud centrado en apoyar la gestión basada en la evidencia en el nivel de la atención primaria de salud. Muchas veces, el proceso de estructuración de los programas y políticas públicas se realiza sin acceso a las mejor evidencia científica. En ese contexto, el sistema integrado de información de Mais Médicos (SIMM) que se describe aquí materializó un esfuerzo para suplir la carencia de información para la gestión en materia de salud. EI SIMM, creado con el objetivo de integrar los datos de las principales fuentes de información relativas al proyecto de cooperación entre el Gobierno del Brasil y la Organización Panamericana de la Salud/Organización Mundial de la Salud (OPS/OMS) para la provisión de médicos destinados al programa Más Médicos, se utilizó para optimizar la generación y consolidación de información a fin de sustentar las decisiones, además de servir de apoyo para el control administrativo y financiero. La experiencia ilustra el desarrollo de una solución complementaria que puede ampliarse para observar los parámetros de recursos humanos en salud no solo en el programa Más Médicos, sino en todo el ámbito de la atención primaria de salud. La pericia adquirida creó condiciones propicias para poder configurar el sistema como un bien público, con miras a ampliar su alcance más allá del apoyo a la gestión de un proyecto específico. Es posible adaptar y reproducir el sistema en otros países de la Región de las Américas para mejorar la disponibilidad y la calidad de la información utilizada por los responsables de tomar decisiones.

Palabras clave Sistemas de información; sistemas de información administrativa; diseminación de información; política informada por la evidencia; atención primaria de salud. 